\title{
Research Article \\ Function Projective Synchronization of a Class of Chaotic Systems with Uncertain Parameters
}

\author{
Junbiao Guan \\ School of Science, Hangzhou Dianzi University, Hangzhou, Zhejiang 310018, China \\ Correspondence should be addressed to Junbiao Guan, junbiaoguan@gmail.com
}

Received 1 December 2011; Accepted 24 February 2012

Academic Editor: Ahmad M. Harb

Copyright (C 2012 Junbiao Guan. This is an open access article distributed under the Creative Commons Attribution License, which permits unrestricted use, distribution, and reproduction in any medium, provided the original work is properly cited.

This paper investigates the function projective synchronization of a class of chaotic systems with uncertain parameters. Based on Lyapunov stability theory, the nonlinear adaptive control law and the parameter update law are derived to make the state of two chaotic systems function projective synchronized. Numerical simulations are presented to demonstrate the effectiveness of the proposed adaptive scheme.

\section{Introduction}

Synchronization of chaotic systems has attracted considerable attention from scientists and engineers and has been investigated intensively both theoretically and experimentally over the last decade. A variety of methods have been introduced for the synchronization of chaotic systems which include complete synchronization, generalized synchronization, phase synchronization, lag synchronization, adaptive synchronization, time scale synchronization, intermittent lag synchronization, projective synchronization, and function projective synchronization, see [1-6] and references therein. Amongst all these synchronization schemes, it is worthwhile to mention that function projective synchronization has attracted much attention recently due to its potential application in secure communications. Function projective synchronization is the more general definition of projective synchronization. As compared with projective synchronization, function projective synchronization means that the master and slave systems could be synchronized up to a scaling function, but not a constant. This feature could be used to get more secure communication in application to secure communications, because it is obvious that the unpredictability of the scaling function in function projective synchronization can additionally enhance the security of communication. Motivated by the aforementioned reasons, this paper investigates function projective synchronization of recently developed chaotic systems with uncertain parameters 
using nonlinear adaptive controller. The rest of this paper is organized as follows. In Section 2, the nonlinear adaptive controller is designed to synchronize the chaotic system. In Section 3, numerical simulations are presented to demonstrate the effectiveness of the theoretical analysis.

\section{Adaptive Control Scheme}

The chaotic system introduced by Liu [7] recently is described by

$$
\begin{aligned}
& \dot{x}_{1}=a\left(x_{2}-x_{1}+x_{2} x_{3}\right), \\
& \dot{x}_{2}=b x_{2}-c x_{1} x_{3}, \\
& \dot{x}_{3}=g x_{2}-h x_{3},
\end{aligned}
$$

where $a, b, c, g, h$ are the system parameters, when $a=1, b=2.5, c=1, g=1, h=4$, the system is chaotic.

We take the system (2.1) as the master system, and the slave system with an adaptive control scheme is given by

$$
\begin{aligned}
& \dot{y}_{1}=a_{1}\left(y_{2}-y_{1}+y_{2} y_{3}\right)+u_{1}, \\
& \dot{y}_{2}=b_{1} y_{2}-c_{1} y_{1} y_{3}+u_{2}, \\
& \dot{y}_{3}=g_{1} y_{2}-h_{1} y_{3}+u_{3},
\end{aligned}
$$

where $a_{1}, b_{1}, c_{1}, g_{1}, h_{1}$ are parameters of the slave system which needs to be estimated, and $u_{1}, u_{2}, u_{3}$ are the nonlinear controllers such that the two chaotic systems are function projective synchronized in the sense that

$$
\lim _{t \rightarrow \infty}\left\|y_{i}-\alpha(t) x_{i}\right\|=0, \quad i=1,2,3
$$

where $\alpha(t)$ is the scaling function.

From system (2.1) and system (2.2), we get the error dynamical system which can be written as

$$
\begin{aligned}
& \dot{e}_{1}=a_{1}\left(y_{2}-y_{1}+y_{2} y_{3}\right)+u_{1}-a \alpha(t)\left(x_{2}-x_{1}+x_{2} x_{3}\right)-\dot{\alpha}(t) x_{1} \\
& \dot{e}_{2}=b_{1} y_{2}-c_{1} y_{1} y_{3}+u_{2}-b \alpha(t) x_{2}-c \alpha(t) x_{1} x_{3}-\dot{\alpha}(t) x_{2} \\
& \dot{e}_{3}=g_{1} y_{2}-h_{1} y_{3}+u_{3}-g \alpha(t) x_{2}+h \alpha(t) x_{3}-\dot{\alpha}(t) x_{3}
\end{aligned}
$$

where $e_{i}(t)=y_{i}(t)-\alpha(t) x_{i}(t), i=1,2,3$.

In order to stabilize the error variables of system (2.3) at the origin, we propose the adaptive control law and the parameter update law for system (2.3) as follows:

$$
\begin{aligned}
& \dot{u}_{1}=-a_{1}\left(y_{2}-y_{1}+y_{2} y_{3}\right)+\dot{\alpha}(t) x_{1}+a_{1} \alpha(t)\left(x_{2}-x_{1}+x_{2} x_{3}\right)-k_{1} e_{1} \\
& \dot{u}_{2}=-b_{1}\left(y_{2}-x_{2} \alpha(t)\right)+c_{1}\left(y_{1} y_{3}+x_{1} x_{3} \alpha(t)\right)+\dot{\alpha}(t) x_{2}-k_{2} e_{2}, \\
& \dot{u}_{3}=-g_{1}\left(y_{2}-x_{2} \alpha(t)\right)+h_{1}\left(y_{3}-x_{3} \alpha(t)\right)+\dot{\alpha}(t) x_{3}-k_{3} e_{3}
\end{aligned}
$$


and the update rules for the five uncertain parameters $a_{1}, b_{1}, c_{1}, g_{1}$, and $h_{1}$ are

$$
\begin{aligned}
& \dot{a}_{1}=\left(x_{1}-x_{2}-x_{2} x_{3}\right) \alpha(t) e_{1}-k_{4} e_{a} \\
& \dot{b}_{1}=-x_{2} \alpha(t) e_{2}-k_{5} e_{b} \\
& \dot{c}_{1}=-x_{1} x_{3} \alpha(t) e_{2}-k_{6} e_{c} \\
& \dot{g}_{1}=-x_{2} \alpha(t) e_{3}-k_{7} e_{g} \\
& \dot{h}_{1}=x_{3} \alpha(t) e_{3}-k_{8} e_{h}
\end{aligned}
$$

where $k_{i}>0(i=1,2, \ldots, 8)$ and $e_{a}=a_{1}-a, e_{b}=b_{1}-b, e_{c}=c_{1}-c, e_{g}=g_{1}-g, e_{h}=h_{1}-h$.

Theorem 2.1. For the given scaling function $\alpha(t)$, the function projective synchronization between the master system (2.1) and the slave system (2.2) can be achieved if the nonlinear controller (2.5) and the update law (2.6) are adopted.

Proof. Construct the following Lyapunov function:

$$
V=\frac{1}{2} \sum_{i=1}^{3} e_{i}^{T} e_{i}+\frac{1}{2}\left(e_{a}^{2}+e_{b}^{2}+e_{c}^{2}+e_{g}^{2}+e_{h}^{2}\right)
$$

Calculating the time derivative of $V$ along the trajectory of error system (2.4), we have

$$
\begin{aligned}
\dot{V}= & e_{1}(t)\left[a_{1}\left(y_{2}-y_{1}+y_{2} y_{3}\right)+u_{1}-a \alpha(t)\left(x_{2}-x_{1}+x_{2} x_{3}\right)-\dot{\alpha}(t) x_{1}\right] \\
& +e_{2}(t)\left[b_{1} y_{2}-c_{1} y_{1} y_{3}+u_{2}-b \alpha(t) x_{2}-c \alpha(t) x_{1} x_{3}-\dot{\alpha}(t) x_{2}\right] \\
& +e_{3}(t)\left[g_{1} y_{2}-h_{1} y_{3}+u_{3}-g \alpha(t) x_{2}+h \alpha(t) x_{3}-\dot{\alpha}(t) x_{3}\right] \\
& +e_{a}(t)\left[\left(x_{1}-x_{2}-x_{2} x_{3}\right) \alpha(t) e_{1}-k_{4} e_{a}\right]+e_{b}(t)\left[-x_{2} \alpha(t) e_{2}-k_{5} e_{b}\right] \\
& +e_{c}(t)\left[-x_{1} x_{3} \alpha(t) e_{2}-k_{6} e_{c}\right]+e_{g}(t)\left[-x_{2} \alpha(t) e_{3}-k_{7} e_{g}\right] \\
& +e_{h}(t)\left[x_{3} \alpha(t) e_{3}-k_{8} e_{h}\right] \\
= & -\mathbf{e}^{T} \mathbf{K e},
\end{aligned}
$$

where $\mathbf{e}=\left[e_{1}, e_{2}, e_{3}, e_{a}, e_{b}, e_{c}, e_{g}, e_{h}\right]^{T}$ and $\mathbf{K}=\operatorname{diag}\left\{k_{1}, k_{2}, k_{3}, k_{4}, k_{5}, k_{6}, k_{7}, k_{8}\right\}$.

It is clear that $\dot{V}$ is negative definite and $\dot{V}=0$ if and only if $\mathbf{e}(t)=\mathbf{0}$. According to the Lyapunov stability theorem, the function projective synchronization is achieved. This completes the proof.

\section{Numerical Simulations}

In this section, we will perform some numerical simulations to verify the effectiveness of the proposed adaptive synchronization controllers. The fourth-order Runge-Kutta method is used to solve the master system (2.1) and the slave system (2.2) with time step size 0.001. The initial conditions of the master system are $x_{1}(0)=0.2, x_{2}(0)=-1.2, x_{3}(0)=0.6$, and those of the slave system are $y_{1}(0)=0.4, y_{2}(0)=0.2, y_{3}(0)=-1.8$. Moreover, the true values of the uncertain parameters are $a=1, b=2.5, c=1, g=1, h=4$, and the initial values of the estimated parameters are chosen as $a_{1}(0)=2, b_{1}(0)=3, c_{1}(0)=3, g_{1}(0)=5, h_{1}(0)=5$, the 

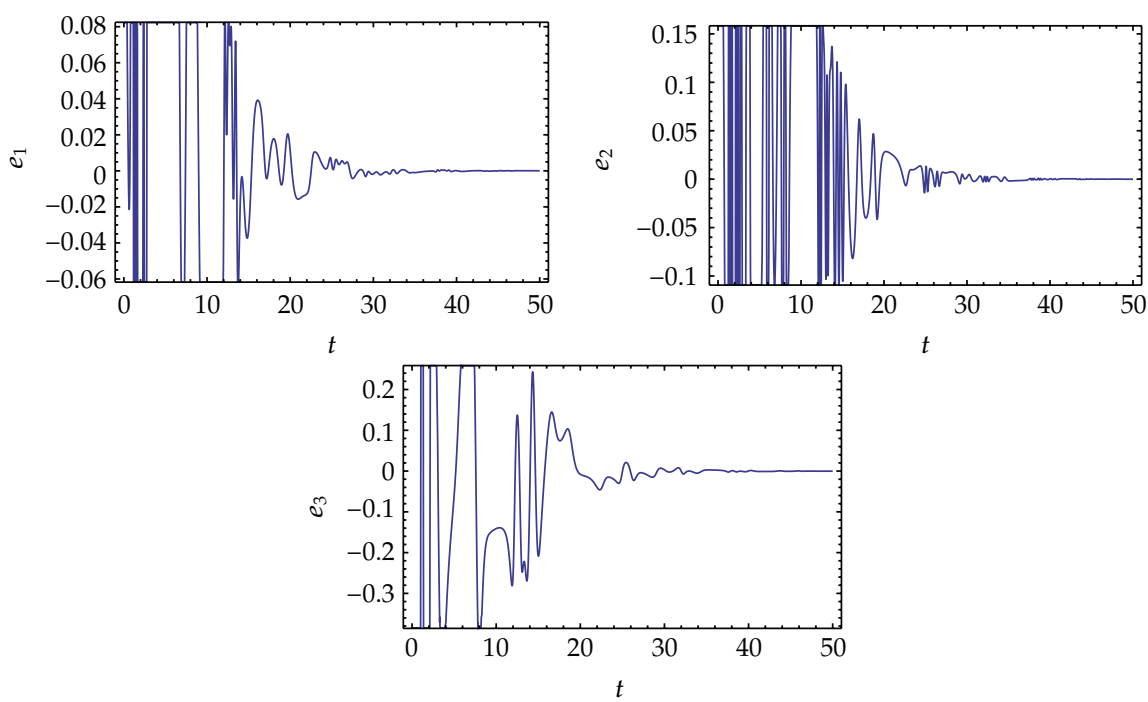

Figure 1: Error dynamics between the master system (2.1) and the slave system (2.2).
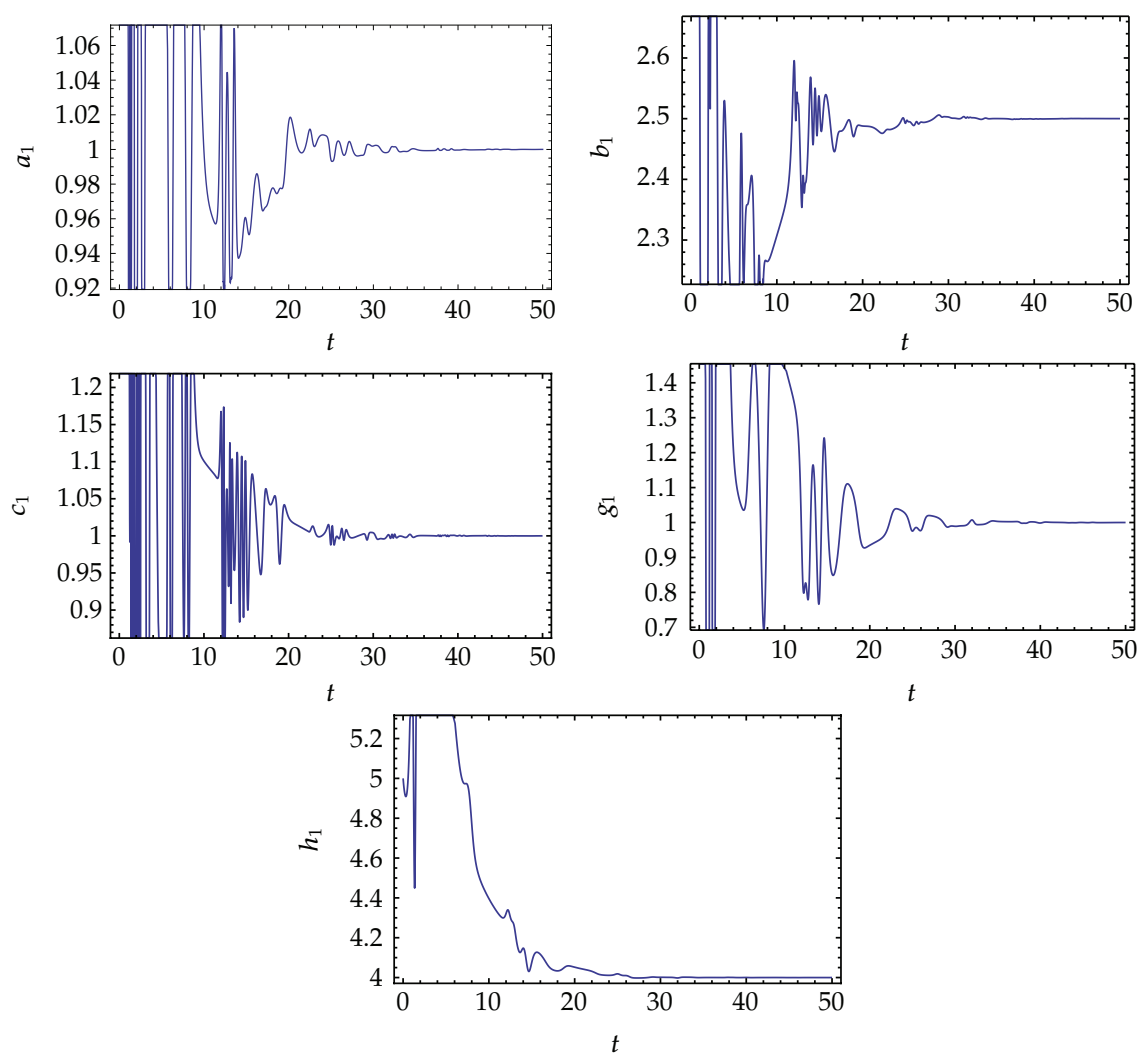

Figure 2: The time evolution of the estimated parameters. 
scaling function is chosen as $\alpha(t)=0.3+0.2 \sin (t)$. Furthermore, the control gains are chosen as $\left(k_{1}, k_{2}, k_{3}, k_{4}, k_{5}, k_{6}, k_{7}, k_{8}\right)=(0.2,0.2,0.2,0.2,0.2,0.2,0.2,0.2)$. The error variables $e_{1}, e_{2}, e_{3}$ which tend to zero with $t \rightarrow \infty$ are illustrated in Figure 1. Figure 2 shows that the estimated values of the uncertain parameters converge to $a=1, b=2.5, c=1, g=1, h=4$ as $t \rightarrow \infty$.

\section{Conclusion}

In summary, this work has investigated the function projective synchronization of the new chaotic systems with uncertain parameters. Based on the Lyapunov stability theory, we have designed the adaptive synchronization controllers with corresponding parameter update laws to stabilize the error dynamics between the master and the slave systems. All the theoretical results are verified by numerical simulations to illustrate the effectiveness of the proposed adaptive function projective synchronization scheme.

\section{References}

[1] L. M. Pecora and T. L. Carroll, "Synchronization in chaotic systems," Physical Review Letters, vol. 64, no. 8 , pp. 821-824, 1990 .

[2] M. Rosenblum, A. Pikovsky, and J. Kurths, "Phase synchronization of chaotic oscillators," Physical Review Letters, vol. 76, pp. 1804-1807, 1996.

[3] H. Taghvafard and G. H. Erjaee, "Phase and anti-phase synchronization of fractional order chaotic systems via active control," Communications in Nonlinear Science and Numerical Simulation, vol. 16, no. 10, pp. 4079-4088, 2011.

[4] R. Mainieri and J. Rehacek, "Projective synchronization in three-dimensional chaotic systems," Physical Review Letters, vol. 82, pp. 3042-3045, 1999.

[5] J. Guan, "Synchronization control of two different chaotic systems with known and unknown parameters," Chinese Physics Letters, vol. 27, Article ID 020502, 2010.

[6] L. Runzi, "Adaptive function project synchronization of Rössler hyperchaotic system with uncertain parameters," Physics Letters. A, vol. 372, no. 20, pp. 3667-3671, 2008.

[7] C. Liu, "A novel chaotic attractor," Chaos Solitons E Fractals, vol. 39, pp. 1037-1045, 2009. 


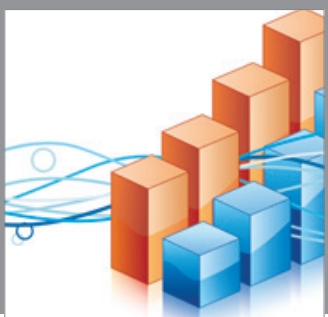

Advances in

Operations Research

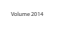

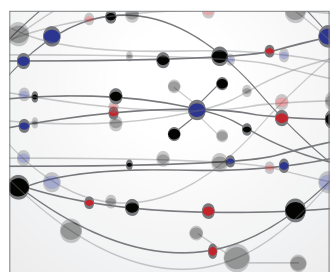

\section{The Scientific} World Journal
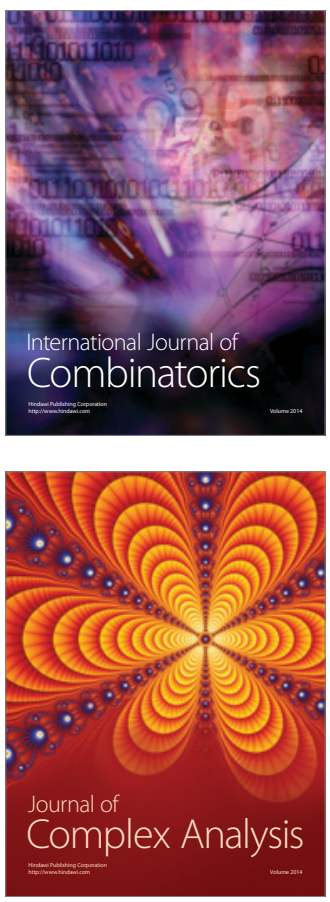

International Journal of

Mathematics and

Mathematical

Sciences
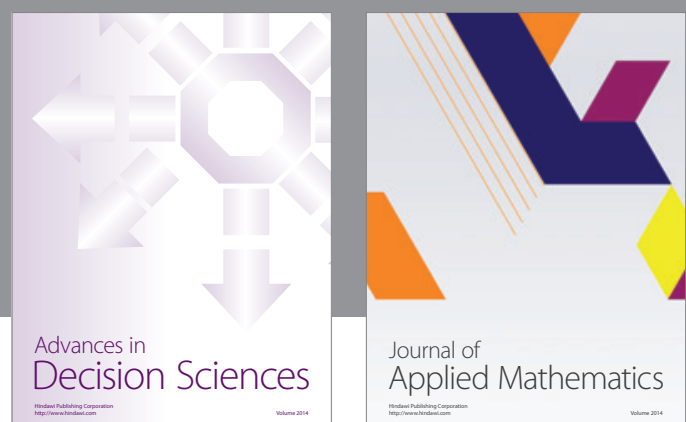

Journal of

Applied Mathematics
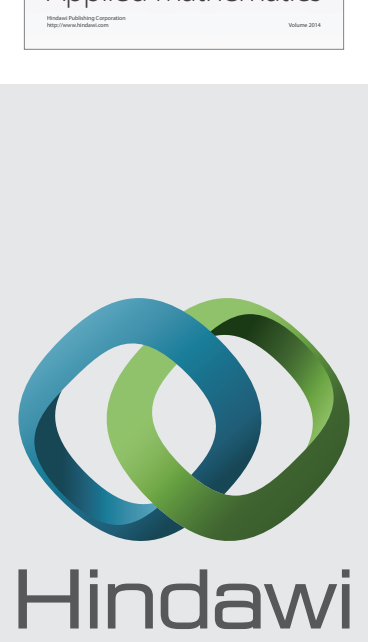

Submit your manuscripts at http://www.hindawi.com
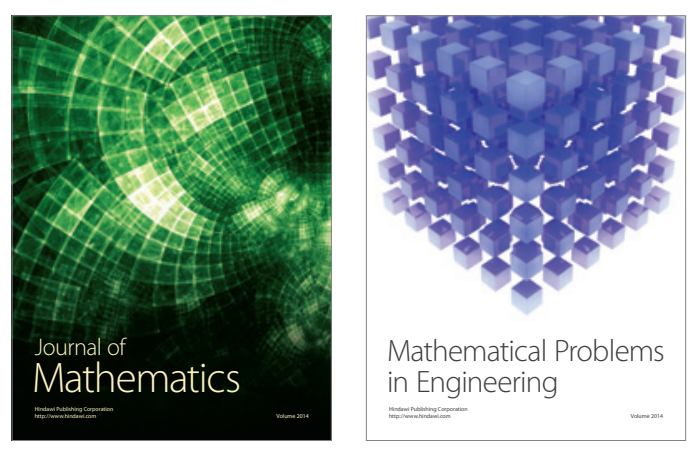

Mathematical Problems in Engineering
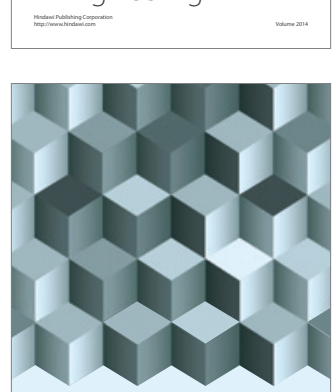

Journal of

Function Spaces
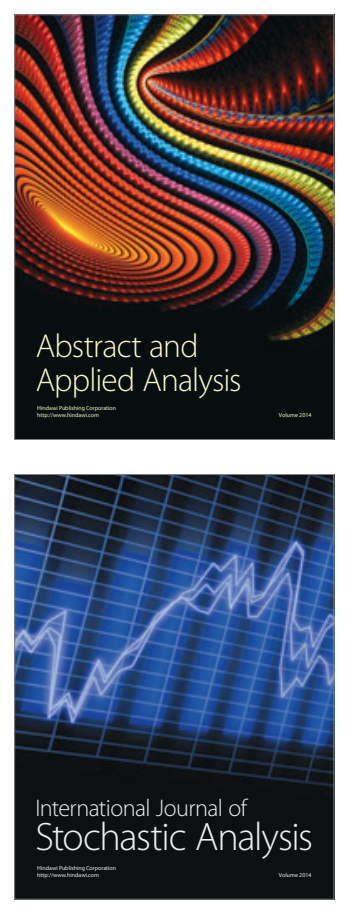

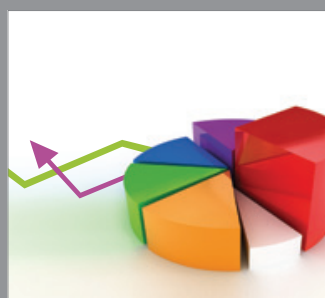

ournal of

Probability and Statistics

Promensencen
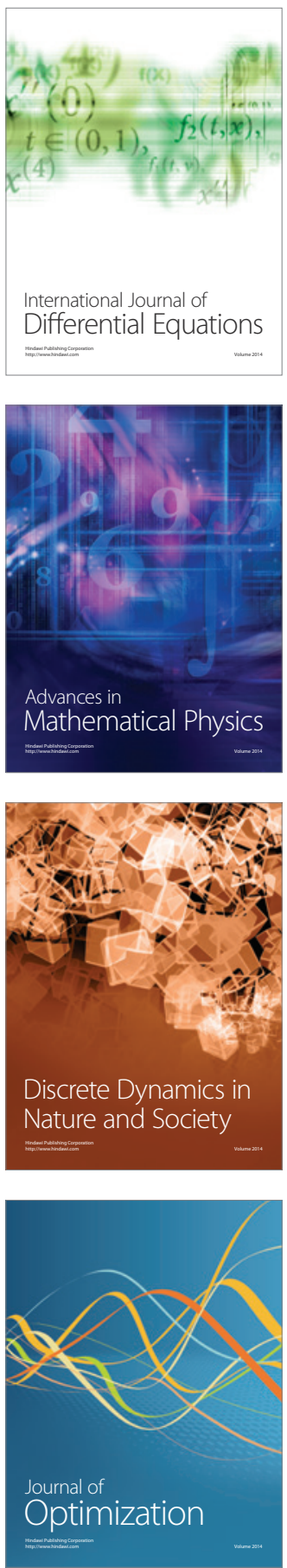